\title{
Design and synthesis of fused polycycles via Diels-Alder reaction and ring-rearrangement metathesis as key steps
}

\author{
Sambasivarao Kotha* and Ongolu Ravikumar
}

\author{
Full Research Paper \\ Address: \\ Department of Chemistry, Indian Institute of Technology-Bombay, \\ Powai, Mumbai-400 076, India, Fax: 022-25767152 \\ Email: \\ Sambasivarao Kotha* - srk@chem.iitb.ac.in \\ * Corresponding author \\ Keywords: \\ Diels-Alder reaction; Grignard addition; ring-rearrangement \\ metathesis; polycycles
}

Beilstein J. Org. Chem. 2015, 11, 1259-1264.
doi:10.3762/bjoc.11.140
Received: 09 April 2015
Accepted: 08 July 2015
Published: 27 July 2015
This article is part of the Thematic Series "Progress in metathesis
chemistry II".
Guest Editor: K. Grela
(C) 2015 Kotha and Ravikumar; licensee Beilstein-Institut.
License and terms: see end of document.

\begin{abstract}
Atom efficient processes such as the Diels-Alder reaction (DA) and the ring-rearrangement metathesis (RRM) have been used to design new polycycles. In this regard, ruthenium alkylidene catalysts are effective in realizing the RRM of bis-norbornene derivatives prepared by DA reaction and Grignard addition. Here, fused polycycles are assembled which are difficult to produce by conventional synthetic routes.
\end{abstract}

\section{Introduction}

Design and synthesis of complex polycycles in a minimum number of steps will enhance the overall synthetic economy of the preparation of a target molecule. The ring-rearrangement metathesis (RRM) is a conceptually novel, synthetically useful atom-economic method for the construction of complex molecules and by this process compounds containing several stereocenters are produced starting from simple starting materials. RRM involves a combination of two or more metathetic transformations, wherein multiple bond forming and bond breaking events take place in a one-pot operation [1-20]. Interestingly, the stereochemical information from the starting material is transferrred to the product. Moreover, RRM enables unprecedented and indirect routes to polycycles. For successful applica- tion of this strategy it is desirable that the starting materials have ring strain so that they can readily undergo a $\mathrm{C}=\mathrm{C}$ double bond cleavage. Release of ring strain is the main driving force for RRM. In this regard, bicyclo[2.2.1] and bicyclo[2.2.2] systems are well suited. Here, we demonstrate that an endocyclic bis-norbornene system undergoes an RRM with a suitably placed olefin moiety to generate complex polycyclic compounds. RRM of norbornene derivatives are common, however, reports dealing with RRM of bis-norbornene derivatives are rare $[21,22]$. Herein, we report two unique examples where the synthesis of hexacyclic systems containing 10 stereocenters have been generated by the application of RRM of readily available bis-norbornene derivatives using Grubbs' catalysts (Figure 1). 


\section{$\mathrm{Cl}_{\mathrm{PCy}}^{\mathrm{Ph}} \mathrm{PCy}_{3}$ \\ Grubbs' $1^{\text {st }}$ generation (G-I) catalyst

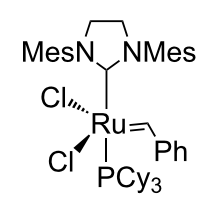 \\ Grubbs' $2^{\text {nd }}$ generation (G-II) catalyst \\ Figure 1: Commercially available ruthenium catalysts used in RRM metathesis.}

The higher analogue related to the bicyclo[2.2.2] system is also studied.

\section{Results and Discussion}

Our strategy to polycycles involves a Diels-Alder reaction (DA) [23-25], a Grignard addition [26] and a RRM as key steps. To begin with, a double DA reaction of cyclopentadiene (1) with 1,4-benzoquinone (2) gave the known bis-adduct 3 $[27,28]$. Later, it was reacted with allylmagnesium bromide to produce 1,2-addition product 4 . A molecular model of compound 3 reveals that its exo-face is more accessible for Grignard addition than the endo-face. Also, the X-ray structure of compound 5 indicates the stereostructure of $\mathbf{4}$. Further, the diol 4 was treated with four equivalents of allyl bromide in the presence of an excess amount of $\mathrm{NaH}$ to generate the mono- $O$-allyl compound $\mathbf{5}$ and surprisingly the di- $O$-allyl compound was not formed. The stereostructure of $\mathbf{5}$ has been established on the basis of single-crystal X-ray diffraction studies [29] and it shows the steric hindrance associated with one of the hydroxy groups (Figure 2).

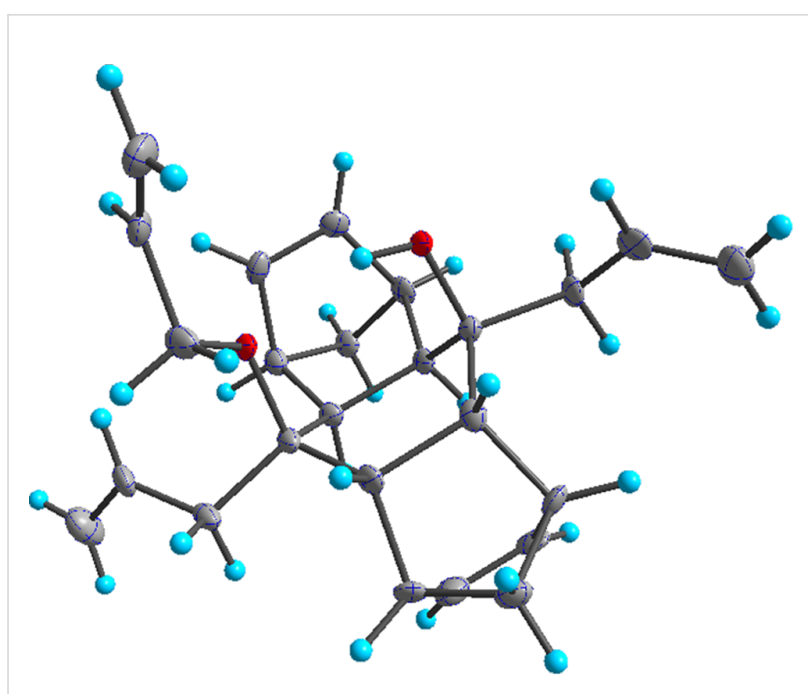

Figure 2: Crystal structure of $\mathbf{5}$ with thermal ellipsoids drawn at $50 \%$ probability level.

Later, the triallyl compound $\mathbf{5}$ was subjected to RRM in the presence of G-II catalyst (Figure 1) under ethylene atmosphere to deliver the hexacyclic rearranged product $6 \mathbf{a}$ in $70 \%$ yield and ring-closing spiro product $\mathbf{6 b}$ in $28 \%$ yield (Scheme 1 ).

To expand this strategy, next we focussed on the preparation of an analogous bicyclo[2.2.2] system and to this end, the DA reaction of 1,3-cyclohexadiene (7) with 1,4-benzoquinone (2) furnished the known bis-adduct 8 [27,28], which on treatment with allylmagnesium bromide delivered diol 9. Later, $O$-allylation of diol 9 with four equivalents of allyl bromide in the presence of $\mathrm{NaH}$ in DMF gave the mono $O$-allyl compound $\mathbf{1 0}$.<smiles>CCO[Sb]([O])(O)C1CC(=O)C=CC1=O</smiles><smiles>O=C1[C@H](C(=O)[C@@H]2C[C@H]3C=C[C@H]2C3)[C@@H]2CC=C[C@H]1C2</smiles>
3<smiles>C=CC[C@]1(O)C2C3C=CC(C3)C2C2C3C=CC(O3)C2C12CC=CCO2</smiles>

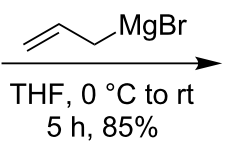

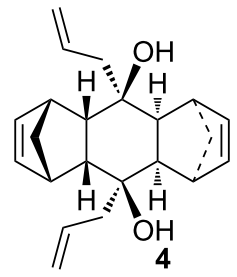<smiles>C=CCBr</smiles>

$\mathrm{NaH}, \mathrm{DMF}$

$1 \mathrm{~h}, 0{ }^{\circ} \mathrm{C}$ to $\mathrm{rt}$ $96 \%$
G-II $(10 \mathrm{~mol} \%)$ ethylene toluene, $70-80^{\circ} \mathrm{C}$

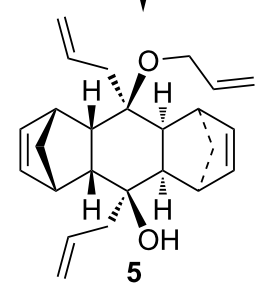


Attempts to achieve complete allylation of $\mathbf{1 0}$ were not successful. Finally, the RRM of compound $\mathbf{1 0}$ in the presence of G-I catalyst (Figure 1) under ethylene atmosphere gave the hexacyclic derivative 11 in $92 \%$ yield (Scheme 2 ). The structures of various polycyclic derivatives have been established on the basis of ${ }^{1} \mathrm{H}$ and ${ }^{13} \mathrm{C}$ NMR spectral data and further supported by HRMS data.

\section{Conclusion}

We have demonstrated a simple, useful and atom-economic methodology for the synthesis of polycycles via DA reaction and RRM as key steps. Here, we generated polycyclic compounds with 10 stereocenters involving six fused rings in four steps starting with readily available starting materials such as 1,3-cyclopentadiene, 1,3-cyclohexadiene and 1,4-benzoquinone. Further studies to expand the scope of this strategy are underway. The strategy demonstrated here is likely to find useful applications in complex targets.

\section{Experimental}

\section{General remarks}

All reactions were monitored by employing thin layer chromatography (TLC) technique using an appropriate solvent system for development. Reactions involving oxygen-sensitive reagents or catalysts were performed in degassed solvents. Dry tetrahydrofuran (THF) and dry ether were obtained by distillation over sodium benzophenone ketyl freshly prior to use. Dichloromethane (DCM) and toluene were distilled over $\mathrm{P}_{2} \mathrm{O}_{5}$ and DMF over $\mathrm{CaH}_{2}$. Sodium sulfate was dried in an oven at $130{ }^{\circ} \mathrm{C}$ for one day. All solvent extracts were washed successively with water and brine (saturated sodium chloride solution), dried over anhydrous sodium sulfate, and concentrated at reduced pressure on a rotary evaporator. Yields refer to the chromatographically isolated sample. All the commercial grade reagents were used without further purification. NMR samples were generally made in chloroform- $d$ solvent, and chemical shifts were reported in $\delta$ scale using tetramethylsilane (TMS) as an internal standard. The standard abbreviations s, d, t, q and $\mathrm{m}$, refer to singlet, doublet, triplet, quartet, and multiplet, respectively. Coupling constants $(J)$ are reported in Hertz.

\section{Experimental procedures Synthesis of compound 4}

Analogously as described in [2], to a stirred solution of diketone $3(0.2 \mathrm{~g}, 0.83 \mathrm{mmol})$ in dry THF $(10 \mathrm{~mL})$ was added allylmagnesium bromide $\left(4.2 \mathrm{~mL}, 1 \mathrm{M}\right.$ solution in ether) at $0{ }^{\circ} \mathrm{C}$ under nitrogen atmosphere, and the reaction mixture was stirred for $5 \mathrm{~h}$ at $\mathrm{rt}$. After completion of the reaction (TLC monitoring), the reaction mixture was quenched with saturated ammonium chloride and extracted with ethyl acetate. The combined organic layer was washed with water, brine and dried over sodium sulfate. The organic layer was concentrated under reduced pressure and the crude product was purified by silica gel column chromatography by eluting with $5 \%$ ethyl acetate in petroleum ether to afford 4 as a white solid $(0.23 \mathrm{~g}, 85 \%)$. mp 130-131 ${ }^{\circ} \mathrm{C}$; ${ }^{1} \mathrm{H}$ NMR (500 MHz, DMSO) $\delta 6.12(\mathrm{~s}, 2 \mathrm{H})$, 6.05-5.98 (m, 2H), $5.92(\mathrm{~s}, 2 \mathrm{H}), 5.14(\mathrm{~d}, J=17.1 \mathrm{~Hz}, 2 \mathrm{H}), 5.06$ $(\mathrm{d}, J=10.2 \mathrm{~Hz}, 2 \mathrm{H}), 4.68(\mathrm{~s}, 2 \mathrm{H}), 2.78(\mathrm{~d}, J=16.8 \mathrm{~Hz}, 4 \mathrm{H})$, $2.48-2.42(\mathrm{~m}, 2 \mathrm{H}), 2.26(\mathrm{~s}, 2 \mathrm{H}), 2.15(\mathrm{dd}, J=14.3,8.3 \mathrm{~Hz}, 2 \mathrm{H})$, $1.61(\mathrm{~s}, 2 \mathrm{H}), 1.19(\mathrm{~d}, J=8.0 \mathrm{~Hz}, 1 \mathrm{H}), 1.11(\mathrm{~d}, J=7.6 \mathrm{~Hz}, 2 \mathrm{H})$,

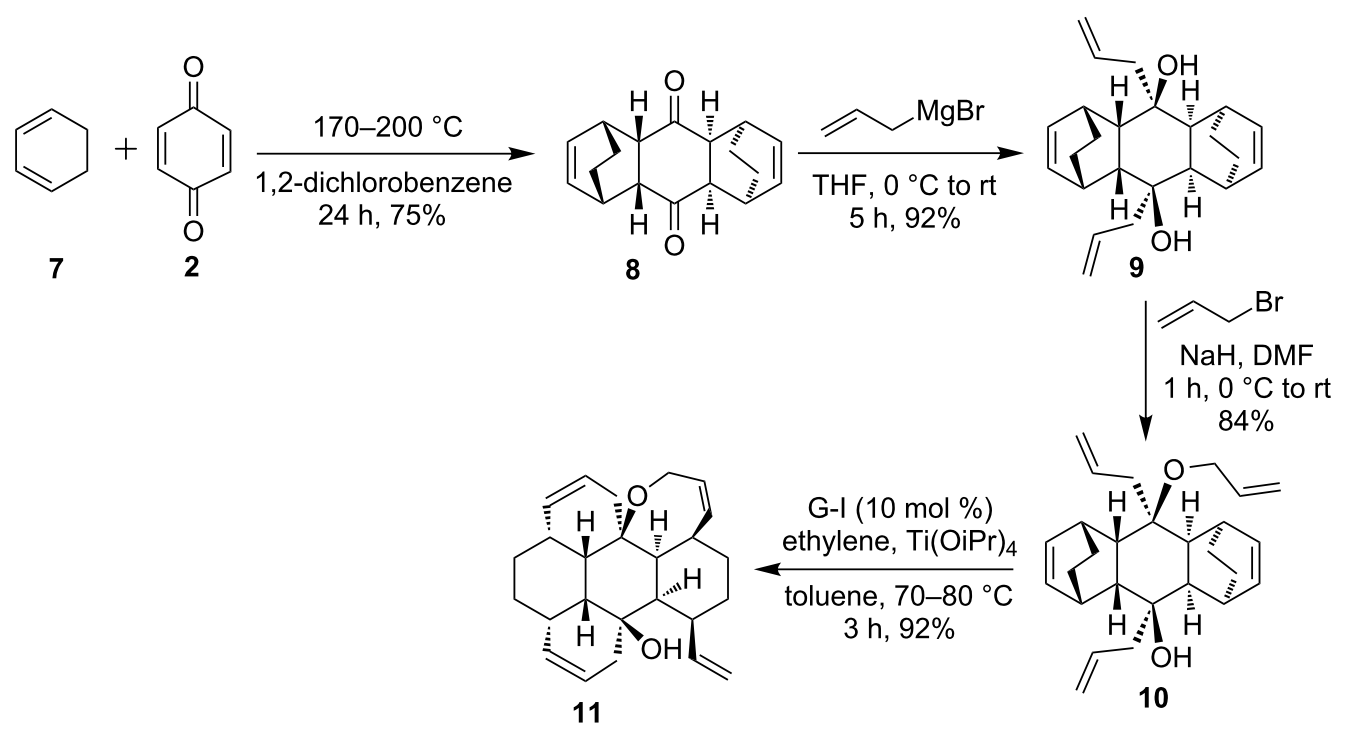


$0.99(\mathrm{~d}, J=7.3 \mathrm{~Hz}, 1 \mathrm{H}) \mathrm{ppm} ;{ }^{13} \mathrm{C} \mathrm{NMR}\left(125 \mathrm{MHz}, \mathrm{CDCl}_{3}\right) \delta$ 135.5, 134.7, 134.4, 118.3, 73.4, 52.3, 52.3, 50.6, 49.2, 45.8, 45.7, $44.8 \mathrm{ppm}$; HRMS (Q-ToF) $m / z:[\mathrm{M}+\mathrm{Na}]^{+}$calcd for $\mathrm{C}_{17} \mathrm{H}_{20} \mathrm{ONa}$, 347.1982; found, 347.1980 .

\section{Synthesis of compound $\mathbf{9}$}

Analogously as described in [2], to a stirred solution of diketone $8(0.5 \mathrm{~g}, 1.8 \mathrm{mmol})$ in dry THF $(10 \mathrm{~mL})$ was added allylmagnesium bromide $\left(11 \mathrm{~mL}, 1 \mathrm{M}\right.$ solution in ether) at $0{ }^{\circ} \mathrm{C}$ under nitrogen atmosphere, and the reaction mixture was stirred for $5 \mathrm{~h}$ at rt. After completion of the reaction (TLC monitoring), the reaction mixture was quenched with saturated ammonium chloride and extracted with ethyl acetate. The combined organic layer was washed with water, brine and dried over sodium sulfate. The organic layer was concentrated under reduced pressure and the crude product was purified by silica gel column chromatography by eluting with $10 \%$ ethyl acetate in petroleum ether to afford 9 as a white solid $(0.6 \mathrm{~g}, 92 \%) . \mathrm{mp}$ $122-125{ }^{\circ} \mathrm{C} ;{ }^{1} \mathrm{H}$ NMR $\left(400 \mathrm{MHz}, \mathrm{CDCl}_{3}\right) \delta 6.29(\mathrm{dd}, J=4.7$, $3.3 \mathrm{~Hz}, 2 \mathrm{H}), 6.18(\mathrm{dd}, J=4.6,3.3 \mathrm{~Hz}, 2 \mathrm{H}), 6.05-5.95(\mathrm{~m}, 2 \mathrm{H})$, $5.16(\mathrm{dd}, J=5.7,1.4 \mathrm{~Hz}, 4 \mathrm{H}), 3.78(\mathrm{~s}, 2 \mathrm{H}), 2.71(\mathrm{~d}$, $J=14.2 \mathrm{~Hz}, 4 \mathrm{H}), 2.62(\mathrm{dd}, J=14.6,6.2,2 \mathrm{H}), 2.26(\mathrm{dd}$, $J=14.6,7.5,2 \mathrm{H}), 2.07(\mathrm{~s}, 2 \mathrm{H}), 1.63(\mathrm{~s}, 2 \mathrm{H}), 1.42(\mathrm{t}, J=6.9 \mathrm{~Hz}$, $4 \mathrm{H}), 1.24-1.21(\mathrm{~m}, 2 \mathrm{H}), 1.15-1.12(\mathrm{~m}, 2 \mathrm{H}) \mathrm{ppm} ;{ }^{13} \mathrm{C}$ NMR $\left(100 \mathrm{MHz}, \mathrm{CDCl}_{3}\right) \delta 134.9,134.2,132.4,117.9,73.7,49.2$, 48.7, 44.7, 32.4, 31.1, 26.6, 26.4 ppm; HRMS (Q-ToF) $m / z$ : $[\mathrm{M}+\mathrm{Na}]^{+}$calcd for $\mathrm{C}_{24} \mathrm{H}_{32} \mathrm{O}_{2} \mathrm{Na}, 375.2295$; found, 375.2293.

\section{Synthesis of compound $\mathbf{5}$}

Analogously as described in [2], to a suspension of $\mathrm{NaH}$ (26 mg, $1.08 \mathrm{mmol})$ in dry DMF $(10 \mathrm{~mL})$, was added solution of compound 4 (50 mg, $0.15 \mathrm{mmol})$ in DMF (5 mL) and allyl bromide $(0.074 \mathrm{~g}, 0.62 \mathrm{mmol})$ at $0{ }^{\circ} \mathrm{C}$ under nitrogen atmosphere and stirred at $\mathrm{rt}$ for $1 \mathrm{~h}$. After completion of the reaction (TLC monitoring), the reaction mixture was quenched with saturated ammonium chloride and extracted with ethyl acetate. The combined organic layer washed with water, brine dried over sodium sulfate. The organic layer was concentrated under reduced pressure and purified by silica gel column chromatography by eluting with $5 \%$ ethyl acetate in petroleum ether to afford 5 as a white solid (60 mg, 96\%). mp 105-108 ${ }^{\circ} \mathrm{C}$; ${ }^{1} \mathrm{H}$ NMR (500 MHz, $\left.\mathrm{CDCl}_{3}\right) \delta 6.09-6.17(\mathrm{~m}, 2 \mathrm{H}), 6.05-5.97$ $(\mathrm{m}, 3 \mathrm{H}), 5.93(\mathrm{dd}, J=5.4,3.0 \mathrm{~Hz}, 1 \mathrm{H}), 5.84-5.76(\mathrm{~m}, 1 \mathrm{H}), 5.22$ $(\mathrm{dq}, J=17.2,1.5 \mathrm{~Hz}, 1 \mathrm{H}), 5.19-5.17(\mathrm{~m}, 1 \mathrm{H}), 5.17-5.07(\mathrm{~m}$, $4 \mathrm{H}), 4.43(\mathrm{~d}, J=1.9 \mathrm{~Hz}, 1 \mathrm{H}), 3.94(\mathrm{dd}, J=11.6,5.7 \mathrm{~Hz}, 1 \mathrm{H})$, $3.85-3.82(\mathrm{~m}, 1 \mathrm{H}), 3.00(\mathrm{~s}, 1 \mathrm{H}), 2.94(\mathrm{~s}, 1 \mathrm{H}), 2.84(\mathrm{~s}, 1 \mathrm{H}), 2.79$ (s, 1H), 2.76-2.70 (m, 1H), $2.65(\mathrm{dd}, J=9.8,3.5 \mathrm{~Hz}, 1 \mathrm{H}), 2.54$ (dd, $J=14.2,6.2 \mathrm{~Hz}, 1 \mathrm{H}), 2.41(\mathrm{dd}, J=9.8,3.5 \mathrm{~Hz}, 1 \mathrm{H}), 2.32$ $(\mathrm{dd}, J=15.6,8.2 \mathrm{~Hz}, 1 \mathrm{H}), 2.18-2.13(\mathrm{~m}, 1 \mathrm{H}), 1.75-1.73(\mathrm{~m}$, $2 \mathrm{H}), 1.42-1.39(\mathrm{~m}, 1 \mathrm{H}), 1.28(\mathrm{~d}, J=4.0 \mathrm{~Hz}, 1 \mathrm{H}), 1.22-1.20(\mathrm{~m}$, $1 \mathrm{H}), 1.0(\mathrm{~d}, J=7.7 \mathrm{~Hz}, 1 \mathrm{H}) \mathrm{ppm} ;{ }^{13} \mathrm{C} \mathrm{NMR}\left(125 \mathrm{MHz}, \mathrm{CDCl}_{3}\right)$ $\delta 135.9,135.3,135.1,134.9,134.4,134.1,133.5,117.0,116.9$, 116.6, 79.4, 72.1, 62.6, 52.4, 51.6, 51.4, 49.6, 49.5, 45.9, 45.7, 45.6, 45.5, 45.1, 44.4, $42.5 \mathrm{ppm}$; HRMS (Q-ToF) $\mathrm{m} / \mathrm{z}$ : $[\mathrm{M}+\mathrm{Na}]^{+}$calcd for $\mathrm{C}_{25} \mathrm{H}_{32} \mathrm{O}_{2} \mathrm{Na}, 387.2295$; found, 387.2295.

\section{Synthesis of compound 10}

Analogously as described in [2], to a suspension of $\mathrm{NaH}$ $(115 \mathrm{mg}, 4.77 \mathrm{mmol})$ in dry DMF $(10 \mathrm{~mL})$, was added a solution of compound 9 (240 $\mathrm{mg}, 0.68 \mathrm{mmol})$ in DMF (10 mL) and allyl bromide $(0.33 \mathrm{~g}, 2.72 \mathrm{mmol})$ at $0{ }^{\circ} \mathrm{C}$ under nitrogen atmosphere and stirred at $\mathrm{rt}$ for $1 \mathrm{~h}$. After completion of the reaction (TLC monitoring), the reaction mixture was quenched with saturated ammonium chloride and extracted with ethyl acetate. The combined organic layer was washed with water, brine and dried over sodium sulfate. The organic layer was concentrated under reduced pressure and purified by silica gel column chromatography by eluting with $5 \%$ ethyl acetate in petroleum ether to afford 11 as a yellow semisolid (224 mg, 84\%). ${ }^{1} \mathrm{H}$ NMR $\left(400 \mathrm{MHz}, \mathrm{CDCl}_{3}\right) \delta 6.22-6.18(\mathrm{~m}, 1 \mathrm{H}), 6.17-6.07(\mathrm{~m}, 3 \mathrm{H})$, 6.07-6.00 (m, 1H), 5.99-5.92 (m, 1H), 5.84-5.73 (m, 1H), 5.31 $(\mathrm{d}, J=2.5 \mathrm{~Hz}, 1 \mathrm{H}), 5.27(\mathrm{dq} J=17.2,1.7 \mathrm{~Hz}, 1 \mathrm{H}), 5.20-5.14$ $(\mathrm{m}, 1 \mathrm{H}), 5.13-5.06(\mathrm{~m}, 3 \mathrm{H}), 3.96-3.87(\mathrm{~m}, 2 \mathrm{H}), 2.80-2.56(\mathrm{~m}$, $7 \mathrm{H}), 2.32(\mathrm{~d}, J=1.5 \mathrm{~Hz}, 1 \mathrm{H}), 2.21-2.14(\mathrm{~m}, 1 \mathrm{H}), 2.40(\mathrm{~d}$, $J=1.6 \mathrm{~Hz}, 1 \mathrm{H}), 1.67(\mathrm{~d}, J=7.0 \mathrm{~Hz}, 2 \mathrm{H}), 1.58(\mathrm{~d}, J=7.5 \mathrm{~Hz}$, $1 \mathrm{H}), 1.57-1.42(\mathrm{~m}, 2 \mathrm{H}), 1.31-1.41(\mathrm{~m}, 2 \mathrm{H}), 1.26-1.11(\mathrm{~m}, 4 \mathrm{H})$ ppm; ${ }^{13} \mathrm{C}$ NMR $\left(100 \mathrm{MHz}, \mathrm{CDCl}_{3}\right) \delta 136.0,135.5,134.3$, 132.9, 132.6, 132.4, 131.9, 117.1, 116.6, 116.4, 79.9, 71.9, 62.4, 50.4, 49.7, 48.6, 44.9, 44.4, 42.9, 32.3, 31.9, 30.7, 30.6, 27.5, 26.7, $26.1 \mathrm{ppm}$; HRMS (Q-ToF) $\mathrm{m} / \mathrm{z}:[\mathrm{M}+\mathrm{Na}]^{+}$calcd for $\mathrm{C}_{27} \mathrm{H}_{36} \mathrm{O}_{2} \mathrm{Na}$, 415.2608; found, 415.2605.

\section{Synthesis of compounds $\mathbf{6 a}$ and $\mathbf{6 b}$}

Analogously as described in [2], to a stirred solution of compound $5(40 \mathrm{mg}, 0.11 \mathrm{mmol})$ in toluene $(40 \mathrm{~mL})$ degassed with nitrogen for 10 minutes, purged with ethylene gas for another 10 minutes and then G-II catalyst ( $8 \mathrm{mg}, 10 \mathrm{~mol} \%$ ) was added and stirred at $70{ }^{\circ} \mathrm{C}$ for $12 \mathrm{~h}$ under ethylene atmosphere. After completion of the reaction (TLC monitoring), the solvent was removed on a rotavapor under reduced pressure and purified by silica gel column chromatography by eluting with 5-10\% ethyl acetate in petroleum ether provided $\mathbf{6 a}$ and $\mathbf{6} \mathbf{b}$ as a colourless liquids (25 mg and $10 \mathrm{mg}, 70 \%$ and $28 \%$, respectively). 6a; ${ }^{1} \mathrm{H}$ NMR $\left(500 \mathrm{MHz}, \mathrm{CDCl}_{3}\right) \delta 6.31-6.24(\mathrm{~m}, 1 \mathrm{H}), 6.10(\mathrm{dd}$, $J=5.6,3.0 \mathrm{~Hz}, 1 \mathrm{H}), 6.05(\mathrm{dd}, J=5.6,2.7 \mathrm{~Hz}, 1 \mathrm{H}), 5.85(\mathrm{dt}$, $J=9.8,2.8 \mathrm{~Hz}, 1 \mathrm{H}), 5.80-5.76(\mathrm{~m}, 1 \mathrm{H}), 5.68-5.65(\mathrm{~m}, 2 \mathrm{H})$, $4.99(\mathrm{dd}, J=17.1,2.4 \mathrm{~Hz}, 1 \mathrm{H}), 4.88(\mathrm{dd}, J=9.9,2.4 \mathrm{~Hz}, 1 \mathrm{H})$, $4.33-4.29(\mathrm{~m}, 1 \mathrm{H}), 4.22-4.18(\mathrm{~m}, 1 \mathrm{H}), 2.95-2.88(\mathrm{~m}, 2 \mathrm{H}), 2.82$ (s, 1H), 2.68-2.61 (m, 2H), $2.56(\mathrm{dd}, J=10.1,3.5 \mathrm{~Hz}, 1 \mathrm{H})$, 2.35-2.29 (m, 1H), 2.21-1.92 (m, 3H), 1.85-1.60 (m, 5H), 1.44 (dt, $J=7.9,1.8 \mathrm{~Hz}, 1 \mathrm{H}), 1.35(\mathrm{~d}, J=8.0 \mathrm{~Hz}, 1 \mathrm{H}) \mathrm{ppm}$; ${ }^{13} \mathrm{C}$ NMR $\left(125 \mathrm{MHz}, \mathrm{CDCl}_{3}\right) \delta=142.5,135.3,132.5,129.2$, 
$123.9,123.8,123.1,114.3,76.2,68.4,60.2,52.3,50.7,49.6$, 47.6, 46.7, 45.9, 42.7, 41.2, 41.1, 40.9, 37.0, 32.3 ppm; HRMS (Q-ToF) $m / z$ : $[\mathrm{M}+\mathrm{Na}]^{+}$calcd for $\mathrm{C}_{25} \mathrm{H}_{32} \mathrm{NaO}_{2}, 359.1982$; found, 359.1988; IR (neat) $v_{\max }: 3050,2954,1691,1610$, $1266 \mathrm{~cm}^{-1}$.

6b; ${ }^{1} \mathrm{H}$ NMR (500 MHz, CD $\left.3 \mathrm{OD}\right) \delta 6.23(\mathrm{dd}, J=5.6,3.0 \mathrm{~Hz}$, $1 \mathrm{H}), 6.17$ (dd, $J=5.7,2.8 \mathrm{~Hz}, 1 \mathrm{H}), 6.15-6.08(\mathrm{~m}, 1 \mathrm{H}), 5.95(\mathrm{t}$, $J=2.4 \mathrm{~Hz}, 2 \mathrm{H}), 5.99-5.89(\mathrm{~m}, 1 \mathrm{H}), 5.72(\mathrm{dd}, J=10.3,2.4 \mathrm{~Hz}$, $1 \mathrm{H}), 5.22(\mathrm{dd}, J=17.1,1.4 \mathrm{~Hz}, 1 \mathrm{H}), 5.14(\mathrm{~d}, J=10.2 \mathrm{~Hz}, 1 \mathrm{H})$, $4.22-4.10(\mathrm{~m}, 2 \mathrm{H}), 2.97(\mathrm{~s}, 1 \mathrm{H}), 2.93(\mathrm{dd}, J=10.0,3.7 \mathrm{~Hz}, 1 \mathrm{H})$, $2.87(\mathrm{~s}, 1 \mathrm{H}), 2.68(\mathrm{~s}, 1 \mathrm{H}), 2.59-2.53(\mathrm{~m}, 2 \mathrm{H}), 2.48(\mathrm{dd}$, $J=10.0,3.4 \mathrm{~Hz}, 1 \mathrm{H}), 2.29(\mathrm{dd}, J=14.4,8.0 \mathrm{~Hz}, 1 \mathrm{H})$, 2.09-2.03 (m, 1H), 1.90-1.84 (m, 2H), 1.43-1.41 (m, 1H), $1.35-1.30(\mathrm{~m}, 4 \mathrm{H}), 1.15(\mathrm{~d}, J=7.6 \mathrm{~Hz}, 1 \mathrm{H}) \mathrm{ppm} ;{ }^{13} \mathrm{C} \mathrm{NMR}$ $\left(125 \mathrm{MHz}, \mathrm{CDCl}_{3}\right) \delta 134.8,134.7,133.9,133.5,124.1,122.6$, 116.0, 73.8, 73.1, 59.9, 51.5, 51.1, 50.9, 49.5, 45.6, 44.6, 44.5, 44.1, 40.1, $32.1 \mathrm{ppm}$; HRMS (Q-ToF) $\mathrm{m} / z$ : $[\mathrm{M}+\mathrm{Na}]^{+}$calcd for $\mathrm{C}_{25} \mathrm{H}_{32} \mathrm{NaO}_{2}$, 359.1982; found, 359.1986 .

\section{Synthesis of compound $\mathbf{1 1}$}

Analogously as described in [2], to a stirred solution of compound $10(20 \mathrm{mg}, 0.05 \mathrm{mmol})$ in toluene $(20 \mathrm{~mL})$ degassed with nitrogen for 10 minutes, purged with ethylene gas for another 10 minutes and then added titanium isopropoxide and G-I catalyst (4 mg, $10 \mathrm{~mol} \%$ ) and stirred at $70-80{ }^{\circ} \mathrm{C}$ for $3 \mathrm{~h}$ under ethylene atmosphere. After completion of the reaction (TLC monitoring), solvent was removed on rotavapor under reduced pressure and purified by silica gel column chromatography by eluting with $5 \%$ ethyl acetate in petroleum ether provided $\mathbf{1 1}$ as a yellow semisolid (17 mg, 92\%). ${ }^{1} \mathrm{H}$ NMR (500 MHz, $\left.\mathrm{CDCl}_{3}\right)$ $\delta 6.24(\mathrm{t}, J=7.1 \mathrm{~Hz}, 1 \mathrm{H}), 6.24-6.11(\mathrm{~m}, 2 \mathrm{H}), 6.07-5.99(\mathrm{~m}$, $2 \mathrm{H}), 5.89-5.84(\mathrm{~m}, 1 \mathrm{H}), 5.63(\mathrm{dq}, J=10.2,2.6 \mathrm{~Hz}, 1 \mathrm{H})$, 5.15-5.08 (m, 3H), 4.19-4.07 (m, 2H), 2.71-2.57 (m, 5H), 2.42-2.34 (m, 2H), 2.24-2.18 (m, 1H), $2.05(\mathrm{~d}, J=10.1 \mathrm{~Hz}$, $1 \mathrm{H}), 1.99-1.94(\mathrm{~m}, 1 \mathrm{H}), 1.62-1.54(\mathrm{~m}, 2 \mathrm{H}), 1.52-1.45(\mathrm{~m}, 1 \mathrm{H})$, $1.44-1.36(\mathrm{~m}, 3 \mathrm{H}), 1.30-1.17(\mathrm{~m}, 4 \mathrm{H}) \mathrm{ppm} ;{ }^{13} \mathrm{C} \mathrm{NMR}$ $\left(125 \mathrm{MHz}, \mathrm{CDCl}_{3}\right) \delta 135.8,133.8,133.1,132.1,131.2,124.9$, 123.4, 116.7, 74.7, 72.5, 61.1, 49.4, 49.2, 49.1, 44.5, 39.6, 34.2, 32.3, 31.5, 31.2, 30.6, 28.1, 27.4, 26.1, 25.3 ppm; HRMS (Q-ToF) $m / z:[\mathrm{M}+\mathrm{Na}]^{+}$calcd for $\mathrm{C}_{25} \mathrm{H}_{32} \mathrm{NaO}_{2}, 387.2295$; found, 387.2292.

\section{Supporting Information}

\section{Supporting Information File 1}

Copies of ${ }^{1} \mathrm{H}$ and ${ }^{13} \mathrm{C}$ NMR spectra of new compounds;

$\mathrm{X}$-ray crystallographic data for compound $\mathbf{5}$.

[http://www.beilstein-journals.org/bjoc/content/

supplementary/1860-5397-11-140-S1.pdf]

\section{Acknowledgements}

We thank the Department of Science and Technology (DST), New Delhi for the financial support and the Sophisticated Analytical Instrument Facility (SAIF), IIT-Bombay for recording spectral data and also thank Gaddamedi Sreevani and Darshan Mhatre for their help in collecting the X-ray data and structure refinement. S. K. thanks the Department of Science and Technology for the award of a J. C. Bose fellowship. O. R. thanks the University Grants Commission, New Delhi for the award of a research fellowship.

\section{References}

1. Kotha, S.; Dipak, M. K. Tetrahedron 2012, 68, 397. doi:10.1016/j.tet.2011.10.018

2. Kotha, S.; Ravikumar, O. Eur. J. Org. Chem. 2014, 5582. doi:10.1002/ejoc.201402273

3. Kotha, S.; Ravikumar, O. Tetrahedron Lett. 2014, 55, 5781. doi:10.1016/j.tetlet.2014.08.108

4. Zuercher, W. J.; Hashimoto, M.; Grubbs, R. H. J. Am. Chem. Soc. 1996, 118, 6634. doi:10.1021/ja9606743

5. Blechert, S.; Holub, N. Chem. - Asian J. 2007, 2, 1064. doi:10.1002/asia.200700072

6. Arjona, O.; Csákÿ, A. G.; Plumet, J. Eur. J. Org. Chem. 2003, 611. doi:10.1002/ejoc.200390100

7. Schmidt, Y.; Lam, J. K.; Pham, H. V.; Houk, K. N.; Vanderwal, C. D. J. Am. Chem. Soc. 2013, 135, 7339. doi:10.1021/ja4025963

8. Lam, J. K.; Pham, H. V.; Houk, K. N.; Vanderwal, C. D. J. Am. Chem. Soc. 2013, 135, 17585. doi:10.1021/ja409618p

9. Standen, P. E.; Kimber, M. C. Tetrahedron Lett. 2013, 54, 4098. doi:10.1016/j.tetlet.2013.05.112

10. Lee, D.; Li, J. Eur. J. Org. Chem. 2011, 4269. doi:10.1002/ejoc.201100438

11. Carreras, J.; Avenoza, A.; Busto, J. H.; Peregrina, J. M. J. Org. Chem. 2011, 76, 3381. doi:10.1021/jo200321t

12. Bose, S.; Ghosh, M.; Ghosh, S. J. Org. Chem. 2012, 77, 6345. doi:10.1021/jo300945b

13. Minger, T. L.; Phillips, A. J. Tetrahedron Lett. 2002, 43, 5357. doi:10.1016/S0040-4039(02)00905-X

14. North, M.; Banti, D. Adv. Synth. Catal. 2002, 344, 694. doi:10.1002/1615-4169(200208)344:6/7<694::AID-ADSC694>3.0.CO;2 $-X$

15. Nguyen, N. N. M.; Leclère, M.; Stogaitis, N.; Fallis, A. G. Org. Lett. 2010, 12, 1684. doi:10.1021/ol100150f

16. Schrock, R. R.; Hoveyda, A. H. Angew. Chem., Int. Ed. 2003, 42, 4592. doi:10.1002/anie.200300576

17. Malik, C. K.; Hossain, Md. F.; Ghosh, S. Tetrahedron Lett. 2009, 50, 3063. doi:10.1016/j.tetlet.2009.04.033

18. Vincent, G.; Kouklovsky, C. Chem. - Eur. J. 2011, 17, 2972. doi:10.1002/chem.201002558

19. Gao, F.; Stamp, C. T. M.; Thornton, P. D.; Cameron, T. S.; Doyle, L. E.; Miller, D. O.; Burnell, D. J. Chem. Commun. 2012, 48, 233. doi:10.1039/C1CC15452D

20. Miege, F.; Meyer, C.; Cossy, J. Org. Lett. 2010, 12, 248. doi:10.1021/ol9025606

21. Malik, C. K.; Ghosh, S. Org. Lett. 2007, 9, 2537. doi:10.1021/ol070906a 
22. Higashibayashi, S.; Tsuruoka, R.; Soujanya, Y.; Purushotham, U.; Sastry, G. N.; Seki, S.; Ishikawa, T.; Toyoto, S.; Sakurai, H. Bull. Chem. Soc. Jpn. 2012, 85, 450. doi:10.1246/bcsj.20110286

23. Nicolaou, K. C.; Snyder, S. A.; Montagnon, T.; Vassilikogiannakis, G. Angew. Chem., Int. Ed. 2002, 41, 1668. doi:10.1002/1521-3773(20020517)41:10<1668::AID-ANIE1668>3.0.CO $; 2-Z$ Angew. Chem. 2002, 114, 1742. doi: 10.1002/1521-3757(20020517)114:10<1742::AID-ANGE1742>3.0.CO; $2-Y$.

24. Kotha, S.; Banerjee, S. RSC Adv. 2013, 3, 7642. doi:10.1039/C3RA22762F

25. Kotha, S.; Misra, S.; Srinivas, V. Eur. J. Org. Chem. 2012, 4052. doi:10.1002/ejoc.201200484

26. Richey, H. G. Grignard Reagents: New Developments; Wiley: Heidelberg, Germany, 2000; pp $418 \mathrm{ff}$.

27. Valiulin, R. A.; Arisco, T. M.; Kutateladze, A. G. Org. Lett. 2010, 12, 3398. doi:10.1021/ol101297b

28. Rathore, R.; Kochi, J. K. J. Org. Chem. 1995, 60, 4399. doi:10.1021/jo00119a017

29. CCDC-1051925 contains the supplementary crystallographic data for this paper. These data can be obtained free of charge from The Cambridge Crystallographic Data Centre (CCDC).

\section{License and Terms}

This is an Open Access article under the terms of the Creative Commons Attribution License

(http://creativecommons.org/licenses/by/2.0), which permits unrestricted use, distribution, and reproduction in any medium, provided the original work is properly cited.

The license is subject to the Beilstein Journal of Organic Chemistry terms and conditions:

(http://www.beilstein-journals.org/bjoc)

The definitive version of this article is the electronic one which can be found at:

doi:10.3762/bjoc. 11.140 